\title{
A Quantitative Model for the Potential Resulting From Reconnection With an Arbitrary Interplanetary Magnetic Field
}

\author{
W. D. Gonzalez and F. S. Mozer
}

Physics Department and Space Sciences Laboratory

University of California, Berkeley, California 94720

\begin{abstract}
A three-dimensional model is utilized to derive the electric potential induced across the magnetosphere by reconnection between the geomagnetic field and an arbitrary interplanetary magnetic field. Assumptions that the magnetosheath flow is uniform and that reconnection is not limited by processes occurring at the magnetopause are made so that the model calculation provides an upper limit to the magnetospheric potential produced by reconnection. The magnitude of the polar cap ionospheric electric field computed from this model is larger than that measured on balloons by an average factor of about 3, and the model reproduces the temporal variations of the experimental data. Some magnetospheric properties related to the magnetopause reconnection line and potential are discussed as a first suggestion toward using the model in studies of relevant magnetospheric problems. The model potential induced across the magnetosphere becomes less dependent on the direction of the interplanetary magnetic field as the strength of its transverse component increases. Thus a reduced correlation between magnetospheric phenomena and the southward component of the interplanetary field should exist when the transverse interplanetary field component exceeds about $8 \gamma$. Observed dependences of the polar cap ionospheric current system on $B_{Y}$ are explained by the model, and similar variations with $B_{X}$ are predicted. Graphs of the model potential as functions of the interplanetary magnetic field components are given as an aid in its application to other problems.
\end{abstract}

Considerable evidence nas accumulated to suggest that many features of magnetospheric dynamics are controlled by the interaction between the interplanetary and terrestrial magnetic fields. Dungey [1961] suggested that the mechanism responsible for this interaction is magnetic field reconnection taking place at the magnetopause, where the two fields meet. The general properties of this interaction are a change in the topology of the reconnecting fields and magnetic energy conversion into thermal and bulk energies of the plasma [Dungey, 1953; Sweet, 1958; Parker, 1963; Petschek, 1964; Yeh and Axford, 1970; Sonnerup, 1972].

Direct evidence for reconnection between the geomagnetic and interplanetary fields arises from observations of energetic solar particles. The first results of this nature were described by Lin and Anderson [1966], and a recent review of subsequent observations has been presented by Morfill and Scholer [1973]. Further experimental evidence has been obtained through correlations of geomagnetic disturbance at ground level with the southward component of the interplanetary magnetic field [Fairfield and Cahill, 1966; Rostoker and Fälthammar, 1967: Schatten and Wilcox, 1967; Nishida, 1968; Arnoldy, 1971], through ground magnetometer correlations with the azimuthal component of the interplanetary magnetic field [Svalgaard, 1968; Mansurov, 1969; Friis-Christensen et al., 1972; Berthelier and Guerin, 1972], through studies of satellite measurements of the tail magnetic field correlated with the southward component of the interplanetary magnetic field [Meng et al., 1973]. through studies of satellite measurements of the inward motion of the magnetopause correlated with the southward component of the interplanetary magnetic field [Meng, 1970; Aubry et al., 1970], and through correlations of electric field measurements with the southward and azimuthal components of the interplanetary magnetic field [Mozer, 1971; Heppner. 1972; Mozer et al., 1974; Mozer and Lucht, 1974].

Most previous studies of magnetic field reconnection have considered only antiparallel reconnecting fields. However, re-

Copyright (c) 1974 by the American Geophysical Union. connection between arbitrarily oriented (other than parallel) fields can occur. Owing to a lack of understanding of the properties of reconnection for such cases, there have been no quantitative calculations of the potential across the magnetosphere (which is a measure of the efficiency of magnetopause reconnection) for an arbitrarily oriented interplanetary field. It is the purpose of this work to present a simplified three-dimensional model for magnetopause reconnection from which a upper limit for the potential across the magnetosphere for arbitrary direction of the interplanetary magnetic field ha been calculated. The major assumptions of this model th result in an overestimate of the magnetospheric potential are 2 nondistorted direction of the solar wind velocity behind the bow shock and a reconnection speed whose magnitude is n limited by physical processes at the magnetopause. The poli cap ionospheric electric field computed from this model ha been compared with that measured on balloons [Mozer ar Gonzalez, 1973] from which it was concluded that the tem poral variations of the measured field were well represented th the model, whereas the average model field exceeded the me sured field by a factor of about 3 .

\section{Description of the Model: Approximations and JUSTIFICATIONS}

Since the interplanetary magnetic field comes into first co tact with the terrestrial magnetic field along the reconnecti line, it is along this line that the interplanetary electric field potential is impressed on the magnetosphere. Hence the $m$ netospheric potential may be calculated by integrating component of the interplanetary electric field along the connection line. To perform this integration, the orientation the reconnection line for an arbitrary interplanetary magne field must be determined. This task will be undertaken in solar-magnetospheric coordinate system and in Gauss units.

The model magnetopause of this calculation is represen as a hemispherical surface of radius $R=15 R_{E}$ with its orig $R_{E}$ tailward of the center of the earth, in agreement 
Alite observations [Fairfield, 1971]. On this magnetopause geomagnetic and interplanetary fields meet and reconnect tinuously with the maximum efficiency allowed by geotric constraints associated with the direction of the internetary field. The geomagnetic field is carried out conuously from inside this surface by the magnetospheric vection that results from the reconnection process. The solar nd flows toward the surface in the $-X$ direction, carrying th it an arbitrarily directed interplanetary magnetic field. Figure $1 a$ illustrates the magnetosheath flow assumed in the del for a purely southward interplanetary magnetic field. e flow within the region of length $L=2 R$ is involved in renection. Depending upon the boundary conditions for the $v$, the interplanetary region associated with reconnection $y$ actually have a dimension $L$ that is less than $2 R$, as is ilrated in Figure $1 b$. Since the electric potential capable of uencing the magnetosphere is the product of the internetary electric field and the distance $L$, this overestimate of n the model probably yields the main contribution to the restimate of the electric potential across the magnetohere. Figure $1 c$ is an illustration of the flow in a closed model which the potential drop along the magnetopause is zero esize of the region $L$ for nonsouthward interplanetary fields ll be computed in the model of the following sections. It is assumed that the day side magnetopause is the only gnetopause region where reconnection takes place. This asmption is reasonable because reconnection should occur $y$ where this process would aid the solar wind flow around magnetospheric obstacle (V. Vasyliunas, private comunication, 1973). At regions where the flow is nearly tangento the magnetopause, namely, outside the hemispherical gion, there is no further need for reconnection, since the lar wind has already passed the obstacle.
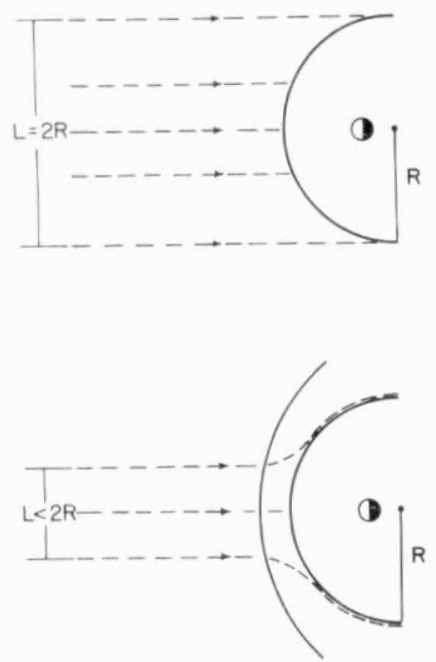

(b)

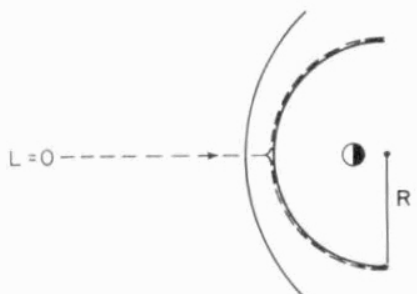

(c)

Fig. 1. Magnetosheath flow configurations with different effective lengths for the interplanetary region in contact with the netopause: $(a) L=2 R$; (b) $0<L<2 R$; (c) $L=0$.
The magnetosheath magnetic field has been approximated as the transverse component of the interplanetary magnetic field, $\mathbf{B}_{T}=\left(B_{Y}, B_{Z}\right)$, multiplied by an amplification factor. which is discussed and estimated in later sections. The amplification factor accounts for the fact that the transverse component of the interplanetary magnetic field is observed [Fairfield, 1967] and expected theoretically [Lees, 1964] to be amplified through the bow shock. Since the electric field associated with reconnection is proportional to $\mathbf{V}_{i} \times \mathbf{B}_{i}$, where $\mathrm{V}_{t}$ $=V_{i} X$ is the solar wind velocity and $\mathbf{B}_{i}=\left(B_{X}, B_{T}\right)$ is the interplanetary magnetic field vector, the $B_{X}$ component of the interplanetary field does not influence the interplanetary electric field. Also, since the major effect of $B_{X}$ on the reconnection geometry might be the translation of the reconnection line along the hemispherical magnetopause and since the magnetopause potential would not be altered appreciably by this translation, the $B_{X}$ component of the interplanetary magnetic field is assumed to be unimportant in the model. This assumption is validated by the lack of dependence on $B_{X}$ of the difference between the model electric field and that measured on balloons [Mozer and Gonzalez, 1973].

\section{Properties of THE ReconNection Line for Nonantiparallel InCident Magnetic FieldS}

The properties of an $X$-type magnetic neutral point were studied initially by Dungey [1953]. He concluded that large currents can exist in a plasma at such points without being opposed by the electromagnetic force, $(1 / c) \mathbf{j} \times \mathbf{B}$, and the neighborhood of such points is unstable with respect to the growth of the current. Several models have been developed to describe the steady state magnetic field reconnection at $X$-type neutral points for two-dimensional systems [Sweet, 1958; Parker, 1963; Petschek, 1964; Yeh and Axford, 1970; Sonnerup, 1972] Figure 2 shows the flow $\mathbf{V}$ and the magnetic field $\mathbf{B}$ configurations around an $X$-type neutral point. The plasma inflow takes place in the $\pm x$ direction. Pairs of magnetic field lines, such as $a b$ and $c d$ (identified via the plasma frozen to them), flow toward the magnetic neutral point. At that point

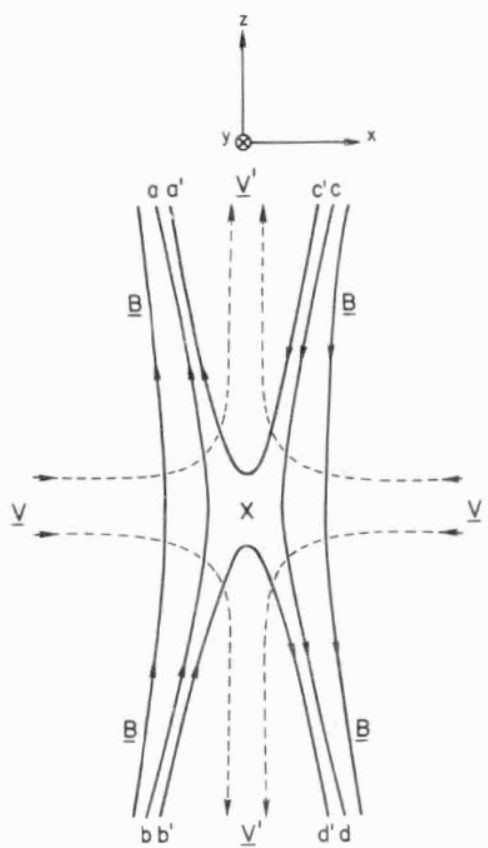

Fig. 2. Flow and magnetic field configuration around an $X$-type neutral point. 
the field lines reconnect to form new pairs of lines, $a^{\prime} c^{\prime}$ and $b^{\prime} d^{\prime}$, in the exit flow. The bulk of the plasma is accelerated to the ejection flow speed via magnetohydrodynamic waves. The frozen field condition is valid everywhere except in the vicinity of the $X$-type neutral point, in which resistive diffusion of the magnetic field through the plasma takes place. Outside the diffusion region, magnetic flux is transported by convection due to the frozen field condition.

A simple three-dimensional system with antiparallel incident magnetic fields would be a uniform extension of Figure 2 in the $y$ direction. In that case, the neutral point becomes a neutral line or reconnection line that lies parallel to the direction of the current required by $\nabla \times B=4 \pi \mathrm{j} / c$, as is illustrated in Figure $3 a$. This reconnection line has the scale length of the system in the $y$ direction and is characterized by the additional fact that its direction is normal to the inflow and outflow velocities and to the direction of the reconnecting components of the incident fields. These characteristics are assumed to remain invariant for a more general system with nonantiparallel incident magnetic fields. Figure $3 b$ illustrates such a general system for magnetic field reconnection on a plane surface. The fields $\mathbf{B}_{1}$ and $\mathbf{B}_{2}$ approach this surface from either side with velocities $\mathbf{V}_{1}$ and $\mathbf{V}_{2}$ and with an angle $\alpha$ between them. The $y$ axis is selected so that the $y$ components of the two fields are equal. The $z$ components of $\mathbf{B}_{1}$ and $\mathbf{B}_{2}$ are antiparallel and connect with each other along the neutral line. This neutral line is parallel to the $y$ axis, since it is assumed to lie in the plane perpendicular to the velocity vectors and to point in the direction along which the nonreconnecting field components are equal. This latter condition follows from the requirement that the magnetic force parallel to the neutral line be zero in order that the flow be confined to directions perpendicular to the neutral line. Since this force is given by the Maxwell stress tensor as $(\partial / \partial x)\left(B_{x} B_{y}\right)$ for the planar geometry of Figure 3, it is assumed that the component of magnetic field in the direction of the neutral line is continuous across the boundary (V. Vasyliunas, private communication, 1973).

The direction of the reconnection line of Figure $3 b$ is again parallel to the current flowing near the neutral line because the only nonzero component of $\nabla \times \mathbf{B}$ is along the $y$ direction. The reconnection line is also perpendicular to the inflow and outflow velocity vectors and to the direction of the reconnecting components of the incident fields. A geometrical

(a)

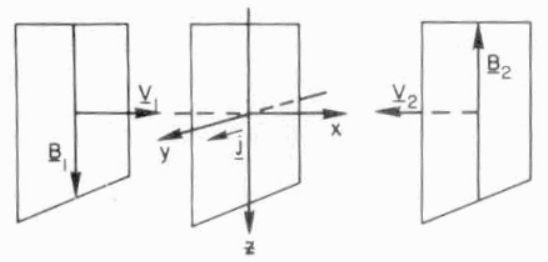

(b)

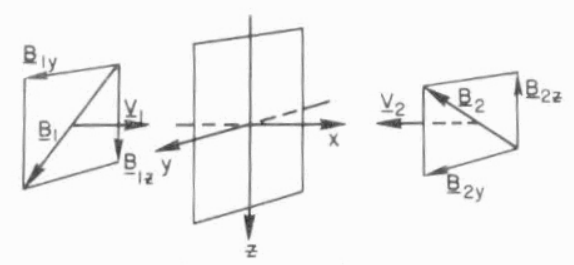

Fig. 3. Three-dimensional magnetic field systems at a plane reconnection boundary with $(a)$ antiparallel fields and $(b)$ nonequal and nonantiparallel fields. The $y$ axis in each drawing is parallel to the reconnection line.

construction giving this direction is obtained from viewing the problem along the $x$ axis, as in Figure $4 a$, and drawing a line joining the heads of the vectors $\mathbf{B}_{1}$ and $\mathbf{B}_{2}$. The perpendicular to this line gives the $y$ direction. The angles that the fields $\mathbf{B}_{1}$ and $\mathbf{B}_{2}$ make with the reconnection line are $\alpha-\beta$ and $\beta$, respectively, and are given from the geometry in Figure $4 a$ as $\sin \beta=\frac{B_{2}-B_{1} \cos \alpha}{\left(B_{1}{ }^{2}+B_{2}{ }^{2}-2 B_{1} B_{2} \cos \alpha\right)^{1 / 2}} \equiv G\left(B_{1} / B_{2}, \alpha\right)$

$\sin (\alpha-\beta)=\frac{B_{1}-B_{2} \cos \alpha}{\left(B_{1}{ }^{2}+B_{2}{ }^{2}-2 B_{1} B_{2} \cos \alpha\right)^{1 / 2}}$

$$
\equiv F\left(B_{1} / B_{2}, \alpha\right)
$$

The condition that reconnection occur for $B_{2} \geq B_{1}$ is $\sin (\alpha$ $\beta)>0$ because only for this case are the $z$ components of the two magnetic fields in Figure $4 a$ antiparallel. Thus the condition for reconnection is

$$
B_{1}>B_{2} \cos \alpha
$$

The case of no reconnection is illustrated by Figure $4 b$ and is given by the condition

$$
B_{1} \leq B_{2} \cos \alpha
$$

Equations (3) and (4) allow the following conclusions. (1) For $B_{2}=B_{1}$, reconnection occurs for any nonzero angle, $\alpha$, between the two magnetic fields. (2) For $B_{2}>B_{1}$, reconnection does not occur for a range of nonzero angles.

From Figures $3 b$ and $4 a$ the components of the fields that reconnect are the antiparallel ones, namely,

$$
B_{12}=B_{1} \sin (\alpha-\beta)
$$

and

$$
B_{2 z}=B_{2} \sin \beta
$$

(a)

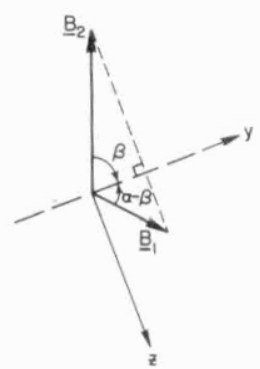

(c)

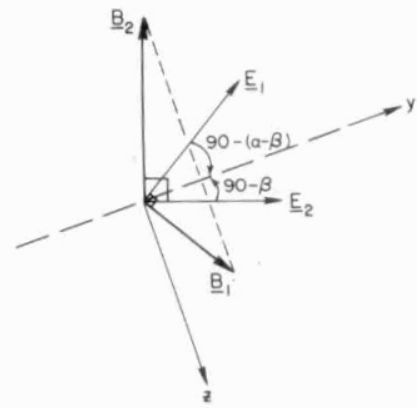

Fig. 4. Diagrams illustrating the reconnection line and the $\alpha$ and $\beta$ for the cases of $(a)$ reconnection and $(b)$ no reconn Diagram $(c)$ illustrates the electric fields. 
For the system illustrated in Figure $3 a$ the potential drop along the reconnection line of length $W$ is

$$
\Phi=\mathrm{E}_{1} \cdot \hat{y} W=\mathrm{E}_{2} \cdot \hat{y} W
$$

where $\hat{y}$ is a unit vector in the $y$ direction and $\mathbf{E}_{1}$ and $\mathbf{E}_{2}$, the convection electric fields at either side of the boundary, are given as

$$
\mathrm{E}_{1}=-(1 / c) \mathbf{V}_{1} \times \mathbf{B}_{1} \quad \mathrm{E}_{2}=-(1 / c) \mathbf{V}_{2} \times \mathbf{B}_{2}
$$

Since $\mathbf{E}_{1}$ and $\mathrm{E}_{2}$ are parallel to the $y$ axis,

$$
\Phi=(1 / c) V_{1} B_{1} W=(1 / c) V_{2} B_{2} W
$$

For the general case illustrated in Figures $3 b$ and $4 c$ the electric fields $E_{1}$ and $E_{2}$ are not parallel to the reconnection line. Therefore after some trigonometric simplification, (7) becomes

$$
\Phi=E_{1} W \sin (\alpha-\beta)=E_{2} W \sin \beta
$$

The terms $\sin (\alpha-\beta)$ and $\sin \beta$ are given by equations (2) and (1), and they will be denoted as the functions $F$ and $G$, respectively, in the remainder of this paper.

By using previous definitions, the potential due to reconnection can be written as

$$
\begin{aligned}
\Phi & =(1 / c) V_{1} B_{1} W F\left(B_{1} / B_{2}, \alpha\right) \\
& =(1 / c) V_{2} B_{2} W G\left(B_{1} / B_{2}, \alpha\right)
\end{aligned}
$$

When $B_{1}=B_{2}, F=G=\sin \alpha / 2$, and the reconnection line becomes the bisector of the reconnecting fields.

\section{Reconnection Line and Potential at the Magnetopause}

The expressions obtained in the previous section will be applied to the magnetosphere with the following identifications. First, $\left|\mathbf{V}_{1} \times \mathbf{B}_{1}\right|=V_{i} B_{T}$, where $V_{i}$ is the interplanetary flow velocity and $B_{T}=\left(B_{Y}^{2}+B_{Z}^{2}\right)^{1 / 2}$ is the transverse component of the interplanetary magnetic field $\mathbf{B}_{i}$. In reality, $\mathbf{V}_{1} \times \mathbf{B}_{1}$ should be given by the magnetosheath quantities $\mathbf{V}_{M}$ and $\mathbf{B}_{M}$. However, because the flow is assumed to be undeviated by the shock or in the magnetosheath, $\mathbf{V}_{i} \times \mathbf{B}_{i}=\mathbf{V}_{M} \times \mathbf{B}_{M}$, and interplanetary values may be used. In later discussions of the quantity $F\left(B_{1} / B_{2}, \alpha\right)$ given by equation (2), $B_{1}$ must be replaced by $B_{M}$.

Second, $\mathbf{B}_{2}=\mathbf{B}_{G}$, where $\mathbf{B}_{G}$ is the geomagnetic field at the hemispherical magnetopause.

Finally, $\alpha$ is the angle between $\mathbf{B}_{G}$ and $\mathbf{B}_{T}, \beta$ is the angle between $\mathbf{B}_{G}$ and the reconnection line, and $W=\pi R$, where $R$ is the radius of the hemisphere.

The major components of the interplanetary magnetic field that influence reconnection are $B_{Y}$ and $B_{Z}$. Thus the geometry of the reconnection line will be obtained under the assumption that $B_{X}=0$. A finite $B_{X}$ causes the reconnection line to be displaced from the nose of the magnetosphere, since the $\mathrm{E} \times \mathrm{B}$ interplanetary flow direction becomes tilted with respect to the sun-earth line. However, the magnitude of this displacement is diminished by the presence of the bow shock, since the postshock flow tends to be oriented more along the sun-earth line than was the interplanetary flow. Since the spherical symmetry of the magnetopause guarantees that the length and angular orientation of the reconnection line do not change greatly due to the displacement of the reconnection line assoaated with finite $B_{X}$, the effect of this component of the interplanetary field in the present model is assumed to be negligible. The discussion of the previous section on the properties of the reconnection line has been given for the case in which the field lines reconnect on a plane surface along which the magnitude and direction of both fields are constant. In the magnetospheric model, however, the reconnection surface is a hemisphere on which the geomagnetic field varies in magnitude and direction. Therefore some geometrical approximations are required to compute the orientation of the reconnection line. For a given value of $\mathbf{B}_{T}$, a magnetic field line touching the nose of the magnetosphere makes an angle $\alpha_{0}$ with the geomagnetic field at the nose, where

$$
\tan \alpha_{0}=\left|B_{Y}\right| / B_{Z} \quad 180^{\circ} \geq \alpha_{0} \geq 0
$$

and $X, Y$, and $Z$ are the conventional rectangular coordinates of the solar-magnetospheric coordinate system. At this point, the reconnection line makes an angle $\beta_{0}$ with the geomagnetic field. Neighboring interplanetary magnetic field lines that approach the hemispherical surface with the same $\mathbf{B}_{T}$ make an angle with the local geomagnetic field that is in general different from that given by (12) for reasons discussed above. Similarly, the angle $\beta$ that the reconnection line makes locally with the geomagnetic field differs from that defined at the nose because $\beta$ depends on $\alpha$. These geometric difficulties will be circumvented by first assuming that the magnetopause reconnection line is obtained as the intersection of the hemisphere with the plane that contains the $X$ axis and that makes an angle $\beta_{0}$ with the noon-midnight meridional plane of the hemisphere.

A view from the sun of the reconnection line geometry is illustrated in Figure 5 for the case of $\alpha$ and $\beta$ constant along the reconnection line and $\alpha_{0}=120^{\circ}$. The interplanetary electric field, $\mathbf{E}_{i}=-\mathbf{V}_{i} \times \mathbf{B}_{T} / c$, is perpendicular to $\mathbf{B}_{T}$ and points in the direction indicated in the figure. Since the line $l$ is perpendicular to $\mathrm{E}_{i}$, it is an electric equipotential, and the potential along the reconnection line is the same as the potential drop in the direction of the interplanetary electric field over the distance $L$. Since $L=2 R F\left(B_{M} / B_{G}, \alpha_{0}\right)$,

$$
\Phi=(2 / c) V_{i} B_{T} R F\left(B_{M} / B_{G}, \alpha_{0}\right)
$$

The limits of the reconnection line on the hemisphere have been assumed to lie at $\varphi= \pm 90^{\circ}$; as a result, the length of the reconnection line is overestimated because its termination at the polar cusps is neglected. This assumption also causes the potential to be overestimated.

In the next section, a more realistic expression for $\Phi$ will be

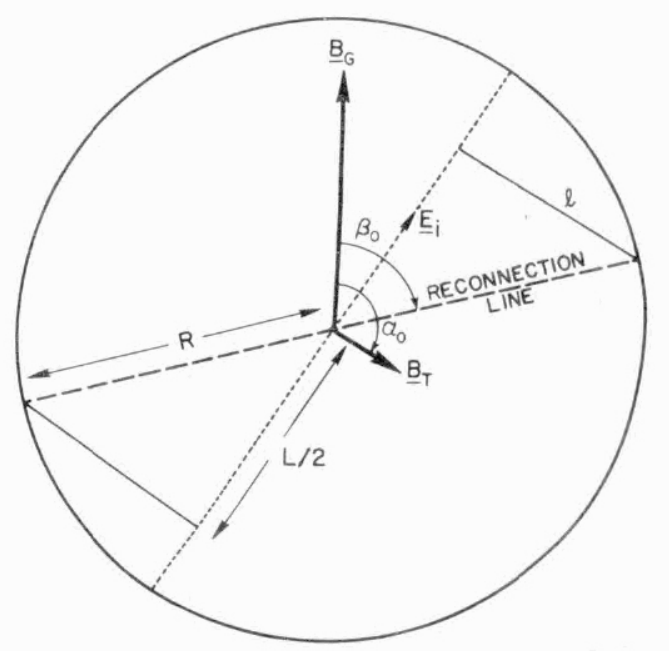

Fig. 5. Reconnection geometry as viewed from the sun. 
obtained, based on better approximations for the geomagnetic and magnetosheath magnetic field magnitudes and geometries.

\section{Quantitative Model for the Potential Due to Magnetopause ReCONNECTION}

In the previous section, $\alpha$ and $\beta$ were assumed to be constant along the reconnection line, which was defined as the intersection of the hemisphere with the plane containing the $X$ axis and making an angle $\beta_{0}$ with the noon-midnight meridional plane. This reconnection line is illustrated as $W_{1}$ in the three-dimensional view of the hemispherical magnetopause given in Figure 6. The intersections of meridional planes containing the dipole axis of the earth with the hemisphere of radius $R$ are indicated in one quadrant of Figure 6 as giving the local direction of $\mathbf{B}_{G}$. For this magnetic field geometry, $\alpha$ increases along $W_{1}$ with increasing distance from the nose. Thus $\beta$ should increase with longitude, since $\beta$ depends on $\alpha$. However, $\mathbf{B}_{G}$ decreases with distance from the nose; as a result, $\beta$ decreases. These two effects approximately cancel, so that the angle $\beta$ between the terrestrial magnetic field and the reconnection line should be approximately constant with longitude. However, because the center of the hemisphere of Figure 6 is $5 R_{E}$ tailward of the origin of the dipole magnetic field, a line on the hemisphere making a constant angle with terrestrial magnetic field lines curves poleward from $W_{1}$ with increasing longitude. Thus the correct reconnection line $W_{2}$ is poleward of $W_{1}$, and hence it lies more along the direction of the interplanetary electric field than does $W_{1}$ (see Figure 5). For this reason, the potential $\Phi_{2}$ along $W_{2}$ is greater than the potential $\Phi_{1}$ along $W_{1}$, which was computed in the last section.

The geometric correction factor $\lambda \equiv \Phi_{2} / \Phi_{1}$ has been calculated numerically on a spherical surface having a magnetopause magnetic field geometry like that given in Figure 5 and a magnetopause field strength given by the Mead [1964] model. The results of this calculation for three magnetosheath field strengths are illustrated in Figure 7. The error bars of this figure include only uncertainties of the numerical procedure utilized to determine the orientation of $W_{2}$. Errors for those

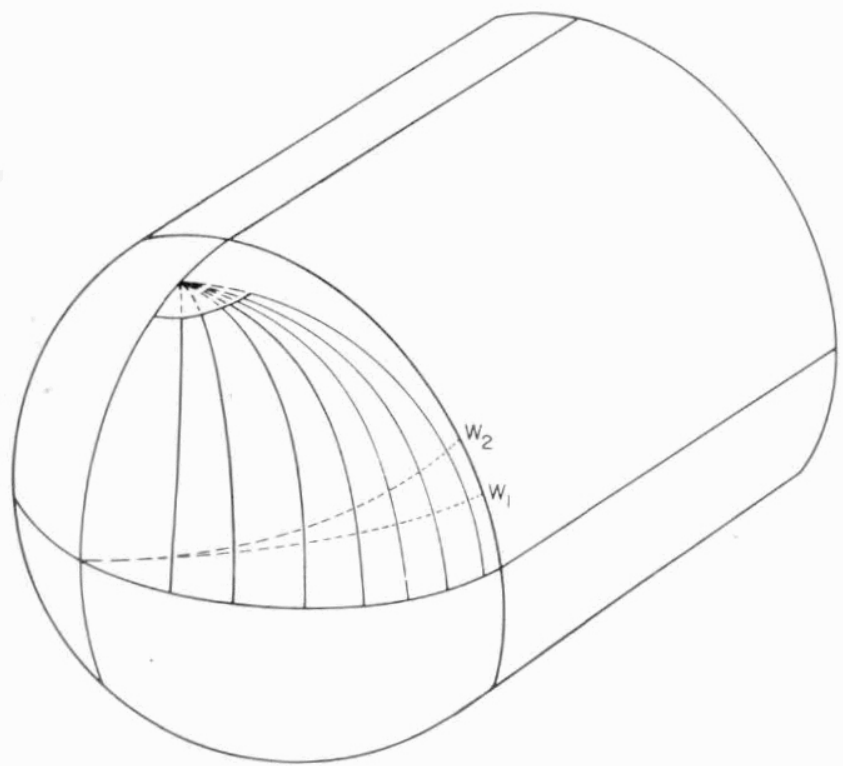

Fig. 6. The geometry of reconnection lines $W_{1}$ and $W_{2}$ on a hemispherical magnetopause on which dipole magnetic field lines are drawn.

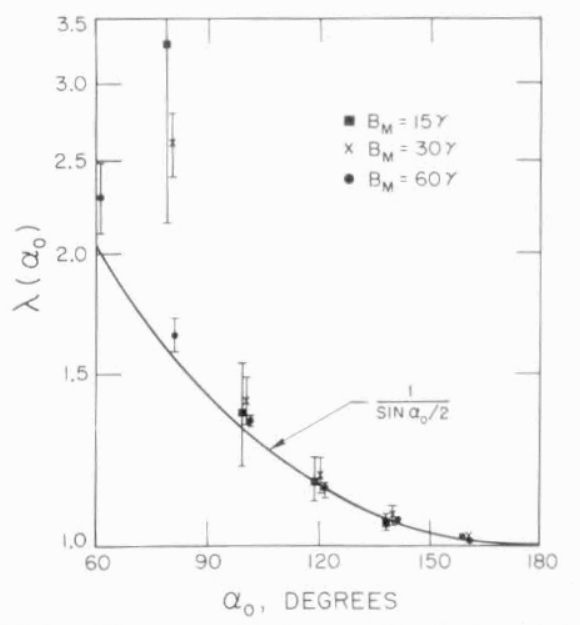

Fig. 7. The geometrical correction factor $\lambda\left(\alpha_{0}\right)$.

data points not having error bars are comparable to the size of the data points. The curve $\lambda\left(\alpha_{0}\right)=\left(\sin \alpha_{0} / 2\right)^{-1}$ fits the numerical estimates at large values of $\alpha_{0}$ with an accuracy comparable to that of the numerical analysis. For small $\alpha_{0}$ the discrepancy between the numerical estimates and the above formula is greater, but this difference is less important both because $\Phi_{1}$ is small and because $\lambda\left(\alpha_{0}\right)$ is overestimated for small $\alpha_{0}$, as is discussed below. Thus a simple correction for the increase of potential due to the poleward shift of the reconnection line in the spherical geometry is $\lambda\left(\alpha_{0}\right)=\left(\sin \alpha_{0} / 2\right)^{-1}$, and the corrected expression for the magnetopause potential becomes

$$
\Phi=\frac{2 V_{i} B_{T} R F\left(B_{M} / B_{G}, \alpha_{0}\right)}{c \sin \alpha_{0} / 2}
$$

Since the function $F$ depends on the magnetosheath field strength $B_{M}$, its calculation from interplanetary data requires estimating the amplification factor $q \equiv B_{M} / B_{T}$. Because the magnetosheath field is observed to be larger than the interplanetary field, an extreme lower limit for $q$ is 1 . Similarly, the magnetosheath field is limited by pressure balance to quirements to less than the geomagnetic field. Therefore an extreme upper limit for $q$ is $B_{G} / B_{T}$, which for normal solar wind conditions $\left(B_{G} \sim 70 \gamma, B_{T} \sim 7 \gamma\right)$ gives $q=10$. Thus

$$
1<q<10
$$

The reasonable value, $q=5$, is assumed in the model, and justification of this value and discussion of effects of other choices are given in the following section. Thus for $B_{G}=70$ ? at the nose of the magnetopause the final expression for the potential given by the model is (14) with

$$
F\left(B_{M} / B_{G}, \alpha_{0}\right)=0
$$

for $\cos \alpha_{0} \geq B_{T} / 14$ and $B_{T}<14$,

$$
\begin{aligned}
& F\left(B_{M} / B_{G}, \alpha_{0}\right)=\frac{B_{T}-14 \cos \alpha_{0}}{\left(B_{T}^{2}-28 B_{T} \cos \alpha_{0}+196\right)^{1 / 2}}(160) \\
& \text { for } \cos \alpha_{0}<B_{T} / 14 \text { and } B_{T}<14 \text {, and } \\
& \qquad F\left(B_{M} / B_{G}, \alpha_{0}\right)=\sin \left(\alpha_{0} / 2\right)
\end{aligned}
$$

for $B_{T} \geq 14$.

Figure 8 gives graphs of (14) and (16) from which the mode polar cap potential may be computed for a range of valus al the $Y$ and $Z$ components of the interplanetary magnetic ficl The value $V_{i}=300 \mathrm{~km} / \mathrm{s}$ has been used for the solar win 


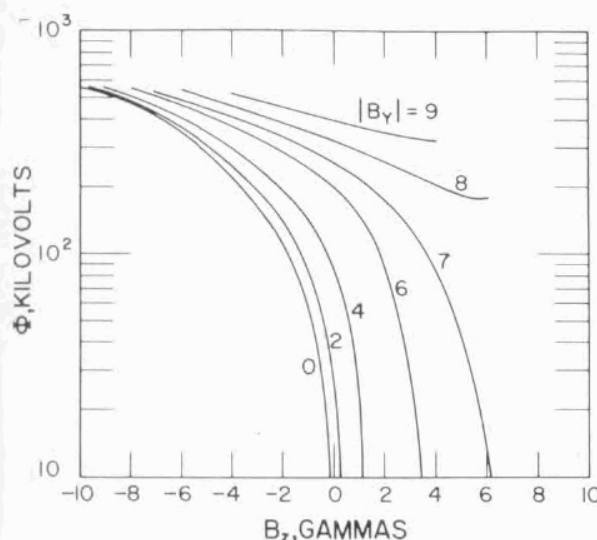

Fig. 8. Electric potential curves for a range of values of the $Y$ and lcomponents of the interplanetary magnetic field. For the best agreeicnt with the experiment, the model potential should be multiplied by

docity in computing the family of curves of Figure 8, and the adius of the magnetopause has been set to $15 R_{E}$. During acre times this radius is smaller, and the solar wind velocity ay be higher. Thus corrections to the potentials of Figure 8 ay be of the order of a factor of 2 during extremely active veriods.

The estimates of Figure 8 and the validity of the present nodel are limited to values of $B_{T}$ less than about $10 \gamma$. This is primarily because $\beta_{0}$ approaches its smallest possible value for igiven $\alpha_{0}\left(\beta_{0} \rightarrow \alpha_{0} / 2\right)$ as the magnetosheath field strength aproaches the magnetopause field strength. Thus the reconnecion line $W_{2}$ becomes more poleward as $B_{T}$ increases. In iality, this poleward displacement is limited by the fact that he immediate postreconnection flow, being perpendicular to the reconnection line, would be opposed to the incident solar wind flow if the reconnection line were located at a high latide. In this case the reconnection line might be displaced uatorward by the incident solar wind flow or else reconnecin might cease because it would not be the easiest mechism for allowing the solar wind to flow around the obstacle. either case the present model overestimates the actual pontial by an amount that probably becomes unrealistically ge for $B_{T}>10 \gamma$. Thus the present calculations have been erminated at this value of the transverse component of the inlerplanetary magnetic field. The effects described above can egin to be discerned at the largest values of $B_{Y}$ in the curves of Figure 8.

\section{Empirical Model for the Potential Due to Magnetopause ReCONNECTION}

A simple means of testing the model calculation of the pointial due to magnetopause reconnection is through its comparison with observations of polar cap ionospheric electric idds, since such fields may be the direct manifestation of the rconnection process. Mozer et al. [1974] have measured ionoipheric polar cap electric fields at Thule, Resolute Bay, and Cambridge Bay for the period of September 3-6, 1971, by using balloon techniques that ae described by Mozer and Serlin 1969]. The magnetic latitudes of Thule, Resolute Bay, and Cambridge Bay are, respectively, $87^{\circ}, 84^{\circ}$, and $78^{\circ}$. From the nalysis of the dawn-to-dusk component of the electric fields observed at Resolute Bay and Cambridge Bay án empirical lue of $3000 \mathrm{~km}$ for the polar cap size has been obtained [Gonzalez, 1973]. The model potential obtained from inter- planetary magnetic field measurements made during the balloon flights (D. H. Fairfield, private communication, 1973) has been divided by this polar cap scale size to obtain a model electric field strength that can be compared with balloon measurements under the assumption that the polar cap potential distribution is uniform. Hourly averages of this computed electric field are plotted in Figure 9 together with the mean of the dawn-to-dusk components of the electric fields observed at Thule and Resolute Bay. The magnitude of the theoretical curve of Figure 9 is larger than the experimental data by an average factor of about 3 , and the time variations of the theoretical and experimental curves are very similar.

In Figure 10 the dawn-to-dusk components of the average measured electric field are plotted against the electric field computed from the model for different ranges of $\alpha_{0}$. The $45^{\circ}$ lines represent the curve expected if the model field were 3 times larger than the measured value. Some part of the spread of the data points in this figure may be attributed to the variable time lag between the presence of an interplanetary magnetic field and its ionospheric manifestation and to the fact that the measured electric field can be negative, whereas the model field cannot. Mean values of the ratio of the measured to model electric field are given in Table 1 for the data of Figure 10, in which $q=5$. Values of the above ratio for $q=2$ and $q=8$ are also given in Table 1. From this table the average ratio of the measured to the computed polar cap electric field for the best choice of $q=5$ is 0.35 . The deviation of this ratio from unity results from the simplifying assumptions in the model that cause an upper limit estimate for the computed electric field. The most important of the assumptions are that the flow to the magnetopause is rectilinear (Figure $1 a$ ) and that there is no limitation imposed on the reconnection rate by the incident flow speed exceeding the Alfvén speed. That the model yields a reasonably small overestimate of the electric field is the strongest justification for these assumptions.

From Table 1 it is also clear that factor of 2 changes in the comparison between the model and experiment can be induced by extreme changes in the value of the amplification factor $q$. In any case, for reasonable values of the amplification factor, the model predicts an upper limit for the magnetospheric potential that exceeds that for the experiment by a factor of about 3. The best empirical fit, using $q=5$, is

$$
\Phi_{\text {empirical }}=0.35 \Phi_{\text {model }}
$$

where $\Phi_{\text {model }}$ is given by the curves of Figure 8 .

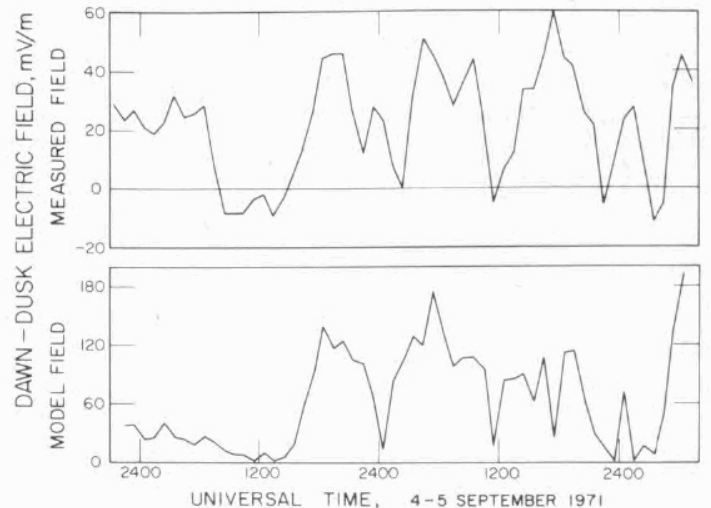

Fig. 9. Hourly averages of the model electric field and the mean of the dawn-to-dusk components of the field measured at Resolute Bay and Thule. The vertical scale of the model field is a factor of 3 greater than that of the measured field. 


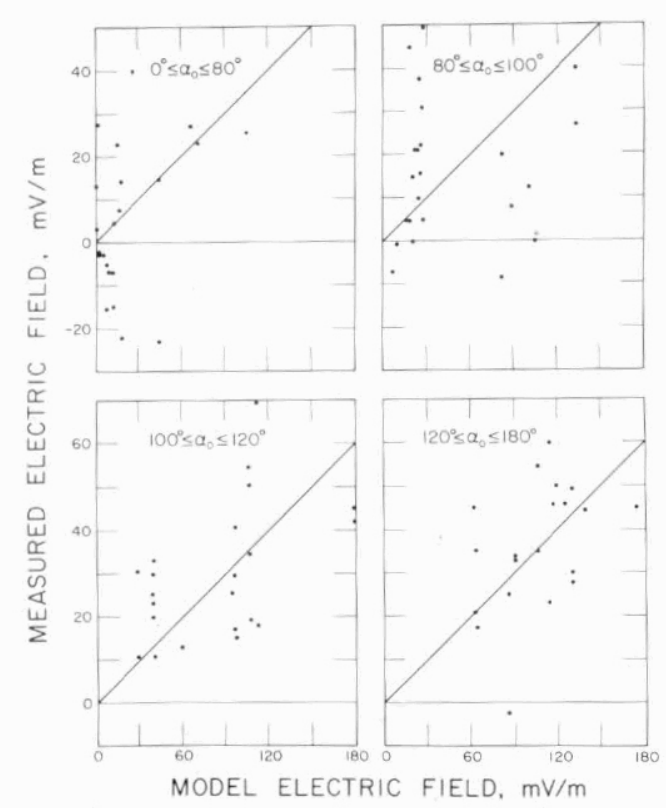

Fig. 10. Hourly averages of the average of the dawn-to-dusk component of the electric fields measured at Thule and Resolute Bay, plotted against the electric field computed from the model for different ranges of $\alpha_{0}$.

The above comparisons of the model potential with balloon electric field measurements require the assumption that the electric field is spatially uniform. Although this assumption may be valid for a large-scale average comparison, some part of the deviation between the model and experiment can be accounted for by the known nonuniformity of the polar cap electric field [Heppner, 1972]. In this regard, it is interesting to note that the model predicts nonuniformities of the kind that are observed because the curvature of the reconnection line $\left(W_{2}\right.$ of Figure 6) implies that most of the potential drop occurs at distances far from the nose. This result follows from the fact that a curved reconnection line is most parallel to the interplanetary electric field direction at large distances from the nose (Figure 5). This nonuniformity will be considered in a later publication.

Another important consequence of the curvature of the reconnection line is that the potential of (14) becomes independent of $\alpha_{0}$ for large $B_{T}$. As $B_{M} \equiv q B_{T}$ approaches $B_{G}, F$ approaches $\sin \alpha_{0} / 2$, and the $\alpha_{0}$ dependence in (14) disappears. This behavior is illustrated in Figure 11 in which model equipotential contours are plotted as functions of $B_{Y}$ and $B_{Z}$. Since the contours tend to become circular with centers at the origin as $\Phi$ increases, the model potential tends toward becoming independent of the direction of the interplanetary magnetic fieid and to depend only on its magnitude. This effect is probably overestimated in the present model because it is related to the poleward extension of $W_{2}$, which is itself overestimated owing to neglect of postreconnection flow effects, as

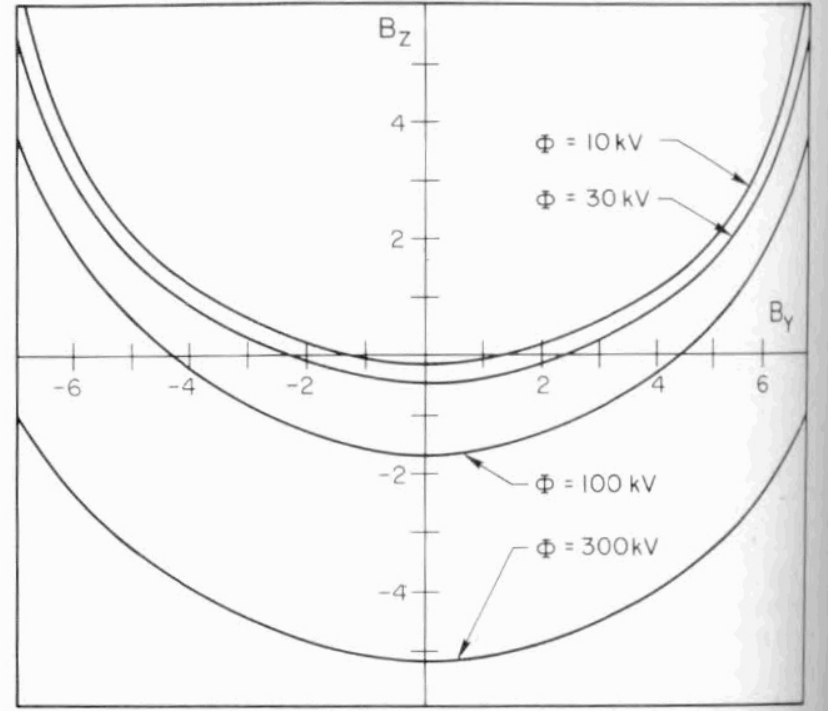

Fig. 11. Equipotential contours of the model electric field plotted as functions of $B_{Y}$ and $B_{Z}$.

was discussed earlier. However, the tendency for the polar cap potential to become independent of the interplanetary mag. netic field direction for large fields must exist in the real problem to complicate correlations between geophysical param. eters and the southward component of the interplanetary magnetic field.

\section{IMPLICATIONS FOR THE MAGNETOSPHERE}

Knowledge of the properties of the reconnection line and of the potential due to magnetopause reconnection allows dis. cussion of some magnetospheric implications. In the model the reconnection line lies in the ecliptic plane when the interplanetary magnetic field is purely southward. The presence of the $Y$ component of the interplanetary magnetic field causes the reconnection line to rotate around the earth-sun axis in clockwise direction if $B_{Y}$ is negative and in the counterclockwise direction if $B_{Y}$ is positive. Since the immediate postro connection flow is normal to the reconnection line, there is thus a tendency for the polar cap flow to occur predominatel over the morning side of the northern hemisphere when $\left.B_{Y}\right\rangle$ and over the afternoon side when $B_{Y}<0$. Thus the $Y$ com ponent of the interplanetary magnetic field causes rotation o the two-cell magnetospheric convection pattern around the sun-earth line that shifts the flow pattern toward dawn (dusk in the northern hemisphere and toward dusk (dawn) in the southern hemisphere when $B_{Y}>0\left(B_{Y}<0\right)$. The cont sequences of the shift of the two-cell convection pattern in the northern hemisphere are the following:

1. The dawn-to-dusk convection electric field over the polar cap has a maximum at local morning (evening) whes $B_{Y}>0\left(B_{Y}<0\right)$.

2. The return flow, associated with the auroral dusk-t

TABLE 1. Ratio of Measured to Computed Electric Fields for Various Ranges of $\alpha_{0}$ and Values of the Amplification Factor $q$

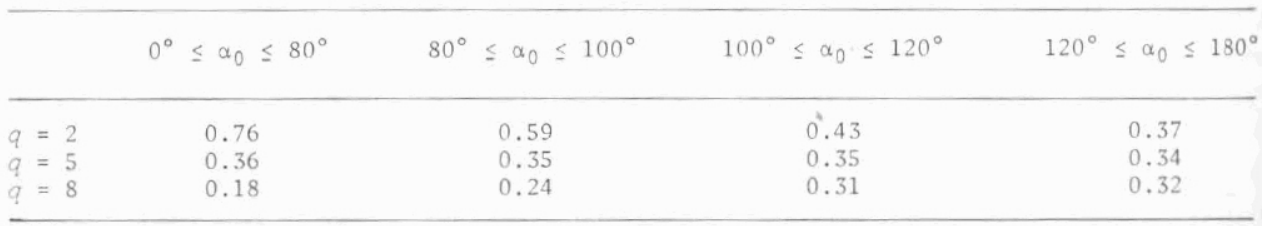


Ln convection electric field, reaches higher latitudes in the string (morning) when $B_{Y}>0\left(B_{Y}<0\right)$.

The ionospheric Hall currents associated with the tilted wcell convection pattern can cause geomagnetic field rations near local noon like those previously observed with pound magnetometers.

Observational evidence for the shift of the two-cell convecin pattern and the three consequences discussed above has en presented [Mozer et al., 1974; Mozer and Lucht, 1974] by ing electric field balloon observations.

Similarly, the direction and magnitude of $B_{X}$ should inlience the location of the polar cap in a way that has not yet wen observed. For example, if $B_{X}<0, B_{Y}=0$, and $B_{Z}>0$, iten interplanetary magnetic field lines are inclined, so that the terplanetary $\mathbf{E} \times \mathbf{B}$ flow approaches the earth from above s northern hemisphere; as a result, the nose of the magnetoause is displaced northward from the equatorial plane. This splacement should cause the ionospheric two-cell convec1 pattern to shift sunward in the southern hemisphere and uiward in the northern hemisphere. Thus when $B_{X} B_{Z}<0$, the orthern hemisphere polar cap should shift tailward and the whern hemisphere polar cap should shift sunward, whereas $B_{x} B_{z}>0$ the opposite should occur. Such effects should be oked for in experimental data.

The power $\Phi I$ dissipated by Joule heating along the magstopause may be estimated for an arbitrary interplanetary magnetic field from the model calculation of the electric poIential and an estimate of the total current $I$ that is obtained fom knowledge of $\nabla \times \mathbf{B} \approx \Delta B / \delta$, where $\Delta B$ is the change of magnetic field across the magnetopause and $\delta$ is the thickness of the magnetopause. For $V_{i}=400 \mathrm{~km} / \mathrm{s}, B_{Y}=6 \gamma$, and $B_{Z}=$ $-4 \gamma$, the power dissipated is $\sim 10^{19} \mathrm{ergs} / \mathrm{s}$ [Gonzalez, 1973]. This energy heats particles near the magnetopause, and such articles precipitate mostly in the polar cusp ionosphere or esape into the interplanetary medium.

Terrestrial magnetic field lines that reconnect at the day side nagnetopause are stretched back by the solar wind to form the magnetospheric tail of length $T$. The energy gained by tretching these field lines is the energy input into the magnetosphere by magnetopause reconnection. The power transferred to the magnetosphere is the integral over the tail of the Poynting flux in the $Z$ direction. Thus

$$
P=\frac{c}{4 \pi} \int d \mathbf{A} \cdot \mathbf{E} \times \mathbf{B}=\frac{c}{4 \pi} \int d X d Y E_{Y} B_{X}{ }^{\prime}
$$

where $E_{Y}$ is the cross-tail electric field and $B_{X}{ }^{\prime}$ is the tail magnetic field. By defining $\int B_{X}{ }^{\prime} d X=\tilde{B} T$, where $\tilde{B}$ is therefore the average tail magnetic field strength, and taking into account the contributions from both hemispheres, the expression for the power transferred to the magnetosphere is given by

$$
P=(c / 2 \pi) \Phi \tilde{B} T
$$

where $\tilde{B}$ may be estimated as the total number of field lines connected on the day side in the time $T / V_{l}$, divided by the half cross-sectional area of the tail, $\pi R^{2} / 2$. Thus

$$
\tilde{B}=\left(2 c \Phi / \pi R^{2}\right)\left(T / V_{i}\right)
$$

From (19) and (20),

$$
P=c^{2} \Phi^{2} T^{2} / \pi^{2} V_{i} R^{2}
$$

The steady state additional energy in the tail due to magetopause reconnection $\mathcal{E}$ is the power $P$ times $T / V_{l}$, the time that it takes for the solar wind to travel the length of the tail. From the above expressions,

$$
\mathcal{E}=c^{2} \Phi^{2} T^{3} / \pi^{2} V_{i}^{2} R^{2}
$$

The length of the tail can be estimated from the following argument [Dungey, 1965]. The time required for the feet of the traveling field lines to cross the polar cap ionosphere, of scale length $d_{1}$ in the sun-to-tail direction, is approximately $d_{1} / V_{e}$, with $V_{e}=c E_{e} / B_{e}$, where $E_{e}$ and $B_{e}$ are the ionospheric polar cap electric and magnetic fields. In the interplanetary medium these field lines move with the solar wind speed $V_{i}$ and spend a time $T / V_{i}$ along the length of the tail. Since these times should be approximately equal,

$$
T \sim V_{i} d_{1} B_{e} / c E_{e}
$$

The ionospheric polar cap electric field $E_{e}$ is given by $\Phi / d_{2}$, where $\Phi$ is the potential due to magnetopause reconnection and $d_{2}$ is the scale length of the ionospheric polar cap in the dawn-to-dusk direction. Therefore

$$
T \sim d_{1} d_{2} V_{i} B_{e} / c \Phi
$$

By using this expression for the length of the tail $T$ in (21) and (22), the power and energy input to the magnetosphere can be written as

$$
\begin{aligned}
& P \sim\left(d_{1} d_{2}\right)^{2} V_{i} B_{e}{ }^{2} / \pi^{2} R^{2} \\
& E \sim\left(d_{1} d_{2}\right)^{3} V_{i} B_{e}{ }^{3} / \pi^{2} R^{2} c \Phi
\end{aligned}
$$

If the dependence of either the lengths of the tail or the polar cap on the magnetospheric potential were known, the above expressions could be used to calculate energy input and storage in the magnetosphere for an arbitrary direction of the interplanetary magnetic field. In the absence of such knowledge, one can obtain typical values of these quantities from empirical data. If the values $d_{1}=d_{2}=3000 \mathrm{~km}, V_{i}=400$ $\mathrm{km} / \mathrm{s}, R=15 R_{E}, B_{e}=0.6 \mathrm{G}$, and $\Phi=50 \mathrm{kV}$ are used, then the length of the tail is $T \sim 600 R_{E}$, the power input to the tail is $P \sim 5 \times 10^{19} \mathrm{ergs} / \mathrm{s}$, and the energy in the tail is $\mathcal{E} \sim 10^{23}$ ergs. Therefore the energy input to the magnetosphere by reconnection is sufficient to power all known magnetospheric processes,

\section{Conclusions}

A quantitative three-dimensional model for the electric potential arising from magnetopause reconnection has been obtained in which several approximations concerned with the configuration of the magnetosheath flow, limitations on the magnitude of the reconnection speed, and geometry of the problem have been made. These approximations are such that the model gives an upper limit for the potential. However, when the ionospheric polar cap electric field computed from the model is compared with that measured on balloons, there is agreement to better than a factor of 3 , as is shown in Figure 9. Therefore the model can be compared to observations of magnetospheric quantities relevant to it, of which polar cap electric fields are the most immediate. The magnetospheric potential is given in Figure 8 for a wide range of the $Y$ and $Z$ components of the interplanetary magnetic field. For best agreement with the experiment, the potential obtained from this figure should be multiplied by the empirical correction factor, 0.35 .

It is concluded that magnetopause reconnection is a highly efficient process that is probably the dominant mechanism 
driving polar cap convection and supplying energy to the magnetosphere. From these and other considerations, it seems that the most efficient way for the solar wind to pass the magnetospheric obstacle is via magnetopause reconnection.

One problem that remains unresolved is the magnetosheath flow configuration when magnetopause reconnection is continuously present. This flow cannot be obtained unless a complete and self-consistent solution is found. Progress in this direction is being made ( $\mathrm{V}$. Vasyliunas, private communication, 1973). The results obtained from the present model suggest that the magnetosheath flow configuration may depart considerably from the closed model solution, especially when the interplanetary magnetic field is large or southward.

Acknowledgments. We would like to express our thanks to $\mathrm{V}$. Vasyliunas, G. Haerendel, and K. A. Anderson for valuable discussions. This research was supported by the Office of Naval Research under contracts N00014-67-A0112-0068 and N00014-69-A02000-0105, by the National Aeronautics and Space Administration under grant NGR 05-020-559, by the Atmospheric Sciences Section of the National Science Foundation under grants GA-31138 and GA$33112 X$, and by the Instituto de Pesquisas Espaciais (INPE), São Paulo, Brasil.

The Editor thanks B. U. Ö. Sonnerup and T. Yeh for their assistance in evaluating this paper.

\section{REFERENCES}

Arnoldy, R. L., Signature in the interplanetary medium for substorms, J. Geophys. Res., 76, 5189, 1971.

Aubry, M. P., C. T. Russell, and M. G. Kivelson, Inward motion of the magnetopause before a substorm, J. Geophys. Res., 75, 7018, 1970.

Berthelier, A., and C. Guerin, Influence of the polarity of the interplanetary magnetic activity at high latitudes, Rep. GRI/NT/87, 11 pp., Groupe de Rech. Ionos., Centre Nat. de la Rech. Sci., SaintMaur, France, 1972.

Dungey, J. W., Conditions for the occurrence of electric discharges in astrophysical systems, Phil. Mag., 44, 725, 1953.

Dungey, J. W., Interplanetary magnetic field and the auroral zones, Phys. Rev. Lett., 6, 47, 1961.

Dungey, J. W., The length of the magnetospheric tail, J. Geophys. Res., 70, 1753, 1965.

Fairfield, D. H., The ordered magnetic field of the magnetosheath, $J$. Geophys. Res., 72, 5865, 1967.

Fairfield. D. H.. Average and unusual locations of the earth's magnetopause and bow shock, J. Geophys. Res., 76, 6700, 1971.

Fairfield, D. H., and L. J. Cahill, Transition region magnetic field and polar magnetic disturbances, J. Geophys. Res., 71, 155, 1966.

Friis-Christensen, E., K. Lassen, J. Wilhjelm, J. M. Wilcox, W. Gonzalez, and D. S. Colburn, Critical component of the interplanetary magnetic field responsible for large geomagnetic effects in the polar cap, J. Geophys. Res., 77, 3371, 1972.

Gonzalez, W. D., A quantitative three-dimensional model for magnetopause reconnections, Ph.D. thesis, Univ. of Calif., Berkeley, 1973.
Heppner, J. P., Polar cap electric field distributions related to the interplanetary magnetic field direction, J. Geophys. Res., 77, 4877. 1972.

Lees, L., Interaction between the solar plasma wind and the geomagnetic cavity, paper presented at the Aerospace Science Meeting. Amer. Inst. of Aeron. and Astronaut., New York, 1964.

Lin, R. P., and K. A. Anderson, Evidence for connection of geomagnetic tail lines to the interplanetary field, J. Geophys. Res., 71. 4213, 1966

Mansurov, S. M., New evidence of a relationship between magnetic fields in space and on earth, Geomagn. Aeron., 9, 622, 1969.

Mead, G. D., Deformation of the geomagnetic field by the solar wind, J. Geophys. Res., 69, 1181, 1964.

Meng, C.-I., Variation of the magnetopause position with substorm activity, J. Geophys. Res., 75, 3252, 1970.

Meng, C.-1., B. Tsurutani, K. Kawasaki, and S.-I. Akasofu, Cross. correlation analysis of the $A E$ index and the interplanetary magnetic field component, J. Geophys. Res., 78, 617, 1973.

Morfill, G. E., and M. Scholer, Study of the magnetosphere using energetic solar particles, submitted to Space Sci. Rev., 1973.

Mozer, F. S., Origin and effects of electric fields during isolated magnetospheric substorms, J. Geophys. Res., 76, 7595, 1971.

Mozer, F. S., and W. D. Gonzalez, Response of polar cap convection to the interplanetary magnetic field, J. Geophys. Res., 78, 6784 1973.

Mozer, F. S., and P. Lucht, The average auroral zone electric field, J. Geophys. Res., 79, 1001, 1974.

Mozer, F. S., and R. Serlin, Magnetospheric electric field measurements with balloons, J. Geophys. Res., 74, 4739, 1969.

Mozer, F. S., W. D. Gonzalez, F. Bogott, M. C. Kelley, and S. Schutz High-latitude electric fields and the three-dimensional interaction between the interplanetary and terrestrial magnetic fields, J. Geophys. Res., 79, 56, 1974.

Nishida, A., Coherence of geomagnetic DP 2 fluctuations with interplanetary magnetic variation, J. Geophys. Res., 73, 5549, 1968

Parker, E. N., The solar-flare phenomenon and the theory of $r 6$. connection and annihilation of magnetic fields, Astrophys. J. Suppl. Ser. 77, 8, 177, 1963.

Petschek, H. E., Magnetic field annihilation, AAS-NASA Symposium on Physics of Solar Flares, NASA Spec. Publ. 50, p. 425, 1964

Rostoker, G., and C.-G. Fälthammar, Relationship between changes in the interplanetary magnetic field and variations in the magnetic field at the earth's surface, J. Geophys. Res., 72, 5853, 1967.

Schatten, K. H., and J. M. Wilcox, Response of the geomagnetic axtivity index $K p$ to the interplanetary magnetic field, J. Geophys. Res. $72,5185,1967$.

Sonnerup, B. U. O.., Magnetic field reconnection and particle acceleration, paper presented at the NASA Symposium on High-Energ Phenomena on the Sun, Goddard Space Flight Center, Greenbeh. Md., Sept. 28-30, 1972.

Svalgaard, L., Sector structure of the interplanetary magnetic field and daily variations of the geomagnetic field at high latitudes, Geophs Pap. R-6, Danish Meteorol. Inst., Copenhagen, 1968.

Sweet, P. A., The neutral point theory of solar flares, Nuovo Cimentil Suppl. 8, Ser. X, 188, 1958.

Yeh, T., and W. I. Axford, On the reconnection of magnetic field line in conducting fluids, 2, J. Plasma Phys., 4, 207, 1970.

(Received September 26, 1973; accepted May 29, 1974.) 